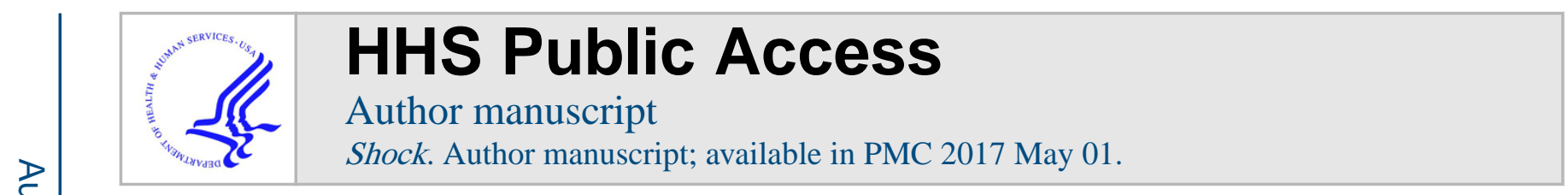

Published in final edited form as:

Shock. 2016 May ; 45(5): 471-474. doi:10.1097/SHK.0000000000000591.

\title{
What's New in Shock, May 2016?
}

Monowar Aziz ${ }^{1}$ and Ping Wang ${ }^{1,2}$

${ }^{1}$ Center for Translational Research, The Feinstein Institute for Medical Research, Manhasset, New York

${ }^{2}$ Department of Surgery, Hofstra Northwell School of Medicine, Manhasset, New York

American novelist Katherine Dunn's famous quote "Prior to penicillin and medical research, death was an everyday occurrence. It was intimate."envisions the advancement of medical research to meet the quality and expectancy of life. The journal Shock has been a pioneer in focusing cutting edge progresses in the fields of trauma, hemorrhage and sepsis to meet $21^{\text {st }}$ century's public health demands. The current issue of Shock features an exclusive collection of clinical and basic science articles from a diverse group of investigators representing several renowned institutions of four continents of the world. The readers of this latest issue of Shock will also have the flavor of two interesting review articles demonstrating a highthroughput screening of current literature resources.

Toward exploring what's new in Shock, the scientific community may discover it to begin with a very informative review article created by Krezalek et al. (1) focusing the fine-tuning aspects of intestinal microbiome and their possible shift into pathogenic biome to govern the course and outcome of sepsis following surgery. This is a well written review article addressing a real-time concern of early versus late mortality in sepsis. This time shift may be a result of prolonged exposure of the host to the virulent pathogens arisen from health promoting gut flora. The article provides valuable information collected from resourceful literature archives and also presents easy illustrating cartoons, which unequivocally depicts the interaction between intestinal microbiome versus pathobiome and their effect on immune system linking to the different phases of sepsis. Thus, the article describing the novel aspects of unavoidable transition of the normal gut microbiome to pathobiome during the course of critical illness may shift the existing paradigm of disease pathogenesis and implicates an interesting field of future studies.

Multiple organ failure (MOF) following shock is a life-threatening clinical problem and its pathophysiology is still under investigation. Altshuler et al. (2) in their superb review article have provided information regarding this need by dissecting the broad subject matter of organ injury. Here, the authors present in a comprehensible way to unveil how the digestive enzymes that are synthesized in the pancreas and discharged into the small intestine as requirement for normal digestion, may play a pivotal role in multiple organ failure in shock. The authors have explained this interesting phenomenon by easy explainable diagrams to demonstrate that under normal condition the digestive enzymes are compartmentalized in the lumen of the intestine by an intact mucosal epithelial barrier. However, if this barrier gets permeable due to ischemic condition, the digestive enzymes escape into the wall of the lumen. These enzymes digest tissues in the mucosa and generate cytotoxic fragments to 
exaggerate injury and inflammation. The digestive enzymes can also escape into the systemic circulation and activate other degrading proteases which clip the ectodomain of surface receptors, leading to an inhibition of receptor function. As a whole, this review article encompasses a solid story which will improve our understating of the mechanism of MOF in shock and also help design novel therapeutic potential for maintaining normal gut homeostasis.

Patients not only die during the initial, hyper-inflammatory phase of sepsis but also at later time-points associated with a prolonged immunosuppressive state. Segre et al. (3) have performed a fascinating ex vivo study using human blood samples from healthy volunteers to establish an optimum condition for the production of a potential biomarker of immunosuppression in sepsis. It is widely known that the reduced ex vivo lipopolysaccharide (LPS) stimulated whole blood pro-inflammatory cytokine release is a hallmark of immunosuppression in critically ill patients despite having several technical limitations influencing the results of the biomarker production from human blood. Here the authors confirm several variables such as the source of LPS, alternate anticoagulants and alternate incubation period to play critical role for different levels of predicted biomarker production and urge upon the requirement of a standardized and validated technique for biomarker oriented diagnosis of immunosuppressive conditions in sepsis. In support of their findings, the investigators have also provided an informative literature review demonstrating the heterogeneity in methodology employed to perform the ex vivo LPS-stimulated whole blood cytokine release assay. Overall, the article is technically sound and generates novel finding to the consequent users of LPS for diagnosing immunosuppressive state.

After a concrete description on two consecutive review articles and a technical study on diagnostic aspects, Hagiwara et al. (4) are the first to take the wheel of our journey toward clinical studies published in this issue of Shock. Here, in a large scale multi-center based retrospective and observational study, the Japanese Association for the Surgery of Trauma investigators have presented the effect of an early aggressive administration of a high ratio of fresh frozen plasma (FFP) to red blood cells (RBC) within the first 6 and $24 \mathrm{~h}$ after admission on mortality in patients with severe, blunt trauma. The surgeons also have provided the survival study findings indicating the patients transfused with an FFP/RBC ratio of $\geq 1$ exhibit a significantly higher rate of survival than the patients transfused with an FFP/RBC ratio of $<1$ within the first $6 \mathrm{~h}$ of admission. Thus, the take home message of this clinical study is quite promising in aspects that it would help implement the approach of early aggressive administration of FFP for the beneficial outcome of patients with severe blunt trauma. In another clinical study published in this issue of Shock by Younan et al. (5) have demonstrated that the spinal cord injury patients with an episode of early coagulopathy depict positive co-relation with an increased susceptibility to ventilator-associated pneumonia. This clinical cohort study is conducted on a decent number of 5-year retrospective patients of spinal cord injury admitted to the level 1 trauma center of the University of Alabama at Birmingham (UAB). The patients' coagulation status is evaluated by standard coagulation test upon arrival, prior to fluid resuscitation, and at $24 \mathrm{~h}$ after admission. The incidence of ventilator associated pneumonia is significantly higher in spinal cord injury patients with coagulopathy, compared with the spinal cord injury patients with no coagulopathy during admission. Consistently, the mortality rate is also shown to be 
significantly higher in spinal cord injury patients with admission coagulopathy than in spinal cord injury patients with no coagulopathy. In contrast, there is no statistical difference in mortality for latent coagulopathy compared with no coagulopathy patients. Although the researchers are aware about the double hit animal models which show increased susceptibility to secondary infection, the current bedside research not only justifies the incidences of hospital-based secondary infection, but also elucidates a novel pathophysiological event, coagulopathy as an independent risk factor for nosocomial infection in hospitalized patients.

The readers can find a decent number of literatures on damage associated molecular patterns (DAMP) in various inflammatory diseases, while majority of those studies are done in animal models. Caro and an esteemed group of investigators (6) have provided an exclusive set of data obtained from an aged matched case-control study to show an increased release of mitochondrial DNA (mtDNA), a DAMP in pediatric sepsis blood samples, which further acts via toll-like receptor-9 to exaggerate injury and inflammation. The investigators have also found that the elevation of mtDNA is associated with an increased severity of illness, and also revealed that there is no difference between plasma mtDNA concentrations in the critically ill non-septic and healthy control patients. Hence, the message is clear to demonstrate the restricted release of mtDNA from pediatric septic children. In association with the clinical finding, a supportive in vitro experiment is designed to show the effect of this potential DAMP in peripheral blood mononuclear cells (PBMC) to release potentially hazardous pro-inflammatory cytokine during sepsis. This easy illustrating and straightforward study will help design novel drugs targeting the DAMP to rescue children from sepsis. In line with the above article on extracellular mtDNA to exacerbate pediatric sepsis, working on this broad field of innate-immune system Andersen et al. (7) in collaboration with a group of Danish investigators in a single center based, prospective pilot study have reported the release of cytochrome $\mathrm{c}$, an unique marker of mitochondrial injury from the adult patients with septic shock. This study not only determines the elevated levels of cytochrome $\mathrm{c}$ in septic patients, but also makes an association between these elevated levels of cytochrome $\mathrm{c}$ with pro-inflammatory markers and poor disease outcomes. Thus, the measurement of extracellular cytochrome c could be an essential prognostic marker is sepsis with extensive mitochondrial damage.

Considerable numbers of studies have been carried-out for prompt identification of cases and early goal-directed therapy for efficient management of severe sepsis and septic shock patients worldwide. Huang et al. (8) from the National Taiwan University Hospital have presented their hospital-based clinical study by recruiting a large number of patients admitted to the intensive care unit for severe sepsis or septic shock from 2007 to 2011 . This is an informative study to update the sepsis demography in their studied septic patients, among them $63 \%$ develop septic shock with an overall mortality rate of about $57 \%$. One important finding has come from their study is that patients who underwent cardiovascular surgery are more likely to experience septic shock after admission and those receiving neurosurgery are at lower risk of developing septic shock. Hence, this article delivers useful information to the physicians regarding risk stratification of septic patients in their country. 
Following an array of clinical studies, the first basic science article in this issue of Shock presented by Sordi et al. (9) has addressed the pathophysiology of multiple organ dysfunction (MOF) in a rat model of hemorrhagic shock (HS). Although it is well-known that the neuronal nitric oxide synthase (nNOS) is implicated in vascular dysfunction and systemic inflammation of septic shock, its role in HS is not adequately understood. The study provides strong in vivo and in vitro data to clarify the role of nNOS in exaggerating tissue injury and inflammation during HS. Here, the investigators have randomly treated hemorrhagic animals with two chemically distinct nNOS inhibitors during the resuscitation period and after four hours several parameters of organ injury and dysfunction are assessed which show that the inhibition of nNOS activity at resuscitation protects rats against MOF and vascular dysfunction. Additionally, the investigators have also provided information regarding the treatment of HS rats with nNOS inhibitors which attenuate neutrophil infiltration into target organs and decrease the activation of NF-kB, inducible NOS expression, nitric oxide production, and nitrosylation of proteins. Overall, this is an ideal translational study where the strategies for targeting nNOS could help ameliorate HSinduced vascular damage and inflammation.

The area focusing the inhibition of $\mathrm{T}$ cell immunosuppression by using neutralizing antibodies against co-inhibitory molecules improves sepsis survival is now being well understood. However, establishing the novel role of T cell co-inhibitory molecules and their ligands in regulating leukocyte migration is unique and should be appreciated. Based on the above theme, Young et al. (10) of a renowned group of shock and critical care medicine fields at the Brown University and Rhode Island Hospital have reported a novel link of the programmed cell death receptor-1 (PD-1) and its ligand PD-L1 expressed in invariant natural killer T cells (iNKT) in promoting their locomotion during inflammation. These investigators have noticed that following experimental sepsis, the iNKT cells emigrate from the liver and into the circulation and peritoneum in a manner dependent upon the coinhibitory molecule PD-1 and PD-L1 interaction. This interaction plays a role in chemotaxis and chemokine receptor expression which in turn facilitates iNKT cell migration. During sepsis, the incidence of neutrophil migration to the site of infection or remote organs has been well documented in numerous studies. However, the current paper focusing the role of PD-1 and PD-L1 interaction for iNKT cell migration emerges a new area of research for elucidation of sepsis pathophysiology and therapeutic intervention.

It has just been discussed in one of the review articles of this Shock issue that the digestive enzymes can be translocated from intestinal lumen during shock to activate systemic proteases which may lead to cleavage of putative receptors and impair vital signal transductions (2). However, the implications of this phenomenon have not previously been investigated using high-throughput approaches. Here, Aletti et al. (11) in a collaborative study utilize a shotgun label-free quantitative proteomic approach to compare the peptidome of plasma samples from healthy and hemorrhagic shock rats. This fascinating study unambiguously verifies the possible role of uncontrolled proteolytic activity in shock. The study utilizes highly sophisticated tools to analyze the peptidome from plasma and reveals 126 circulating peptides in control and 295 in hemorrhagic shock animals, indicating significantly larger proteolytic activity in shock. The identification and characterization of cleaved peptides may rewrite the paradigm of systemic inflammation caused by hemorrhage 
and also protracts to use this scenario in other inflammatory diseases caused by sepsis. Pneumonia is more likely to be fatal in the elderly. Study carried-out by Turnbull et al. (12) demonstrates an ideal double hit model to clarify how poly trauma enhances the susceptibility to develop pneumonia. The investigators have also compared the survival outcomes between young and mature animals with trauma followed by $P$. aeruginosa inoculation. The study is technically sound and depicts several parameters to strongly support their hypothesis that the mature animals are more susceptible to develop pneumonia following injury. Thus, the study could be a hallmark of improving our understanding of the pathogenesis and disease susceptibility in elderly patients.

For optimum prognosis, timing of the administration of research-based treatment modalities has always been a matter of concern. Fukada et al. (13) from Tokyo Women's Medical University, Japan have carried-out an immensely valuable study to set up an optimum timing of morphine administration on LPS-mediated lethal endotoxic shock in mice (13). Here, the investigators evaluate the effect of the timing of morphine administration on the survival rate, histological and several immunological parameters and suggest that morphine treatment before LPS challenge suppresses lethal endotoxic shock, in contrast if morphine is administered after LPS it could exacerbate lethal endotoxic shock. Thus in conclusion, one of the factors that determine whether morphine exacerbates or inhibits infection is the timing of its administration which would further help implement morphine as an effective therapeutic potential when administered at the right time of disease progression. Last but not least, another important study published in this issue of Shock by Leelahavanichkul et al. (14) from Thailand has emerged neutrophil gelatinase associated lipocalin (NGAL) as a promising early diagnostic marker of acute kidney injury (AKI) induced by sepsis, bilateral nephrectomy and bilateral ureter obstruction in mice. Moreover, the investigators also have compared the levels of serum NGAL with that of serum creatinine in a time course experiment in several renal injury model animals and suggest that serum NGAL may serve as an early biomarker for diagnosing renal disease conditions. The article also reveals the association between serum NGAL and pro-inflammatory cytokine as determined by regression analysis. Overall, utilizing a bunch of solid experimental tools, the article provides valuable diagnostic information to help upgrade the understanding of researchers and physicians of nephrology field.

In summary, the current issue of Shock represents fourteen interesting articles, among them two deal with systematic review $(1,2)$, five articles focus on clinical studies utilizing human samples (4-8) and the other seven articles are on basic science aspects (3, 9-14). In context of disease phenotypes, ten articles represent research on sepsis, trauma and pneumonia fields $(1-5,7,8,10,12,13)$, two articles deal with HS model $(9,11)$, one article demonstrates pediatric sepsis patients (6), and the last one represents both sepsis and AKI models (14). Each of these articles of this issue of Shock is extremely valuable and provides distinct message, which can move science to a next level by not only rewriting the disease pathogenesis but also reversing the dead-end therapies against life-threatening diseases to a new direction. Thank you for joining us on this wonderful issue of Shock. 


\section{References}

1. Krezalek MA, DeFazio J, Zaborina O, Zaborin A, Alverdy JC. The shift of an intestinal "microbiome" to a "pathobiome" governs the course and outcome of sepsis following surgical injury. Shock. 45:2016.

2. Altshuler AE, Kistler EB, Schmid-Schönbein GW. Autodigestion: Proteolytic degradation and multiple organ failure in shock. Shock. 45:2016.

3. Segre E, Fullerton JN. Stimulated whole blood cytokine release as a biomarker of immunosuppression in the critically ill: The need for a standardized methodology. Shock. 45:2016.

4. Hagiwara A, Kushimoto S, Kato H, Sasaki J, Ogura H, Matsuoka T, Uejima T, Hayakawa M, Takeda M, Kaneko N, Saitoh D, Otomo Y, Yokota H, Sakamoto T, Tanaka H, Shiraishi A, Morimura N. Can early aggressive administration of fresh frozen plasma improve outcomes in patients with severe blunt trauma? A report by the Japanese association for the surgery of trauma. Shock. 45:2016.

5. Younan D, Lin E, Griffin R, Vanlandingham S, Waters A, Harrigan M, Pittet J-F, Kerby JD. Early trauma-induced coagulopathy is associated with increased ventilator-associated pneumonia in spinal cord injury patients. Shock. 45:2016.

6. Di Caro V, Walko TD III, Bola RA, Hong JD, Pang D, Hsue V, Au AK, Halstead ES, Carcillo JA, Clark RSB, Aneja RK. Plasma mitochondrial DNA - a novel DAMP in pediatric sepsis. Shock. 45:2016.

7. Andersen LW, Liu X, Montissol S, Holmberg MJ, Sulmonte C, Balkema JL, Cocchi MN, Gazmuri RJ, Berg KM, Chase M, Donnino MW. Cytochrome C in patients with septic shock. Shock. 45:2016.

8. Huang C-T, Tsai Y-J, Tsai P-R, Yu C-J, Ko WJ. Severe sepsis and septic shock: Timing of septic shock onset matters. Shock. 45:2016.

9. Sordi R, Chiazza F, Collino M, Assreuy J, Thiemermann C. Neuronal nitric oxide synthase is involved in vascular hyporeactivity and multiple organ dysfunction associated with haemorrhagic shock. Shock. 45:2016.

10. Young JS, Heffernan DS, Chung C-S, Kettenmann ML, Young WA, Guillen VS, Cioffi WG, Ayala A. Effect of PD-1:PD-L1 in invariant natural killer T-cell emigration and chemotaxis following sepsis. Shock. 45:2016.

11. Aletti F, Maffioli E, Negri A, Santamaria MH, De Lano FA, Kistler EB, Schmid-Shonbein GW, Tedeschi G. Peptidomic analysis of rat plasma: Proteolysis in hemorrhagic shock. Shock. 45:2016.

12. Turnbull IR, Ghosh S, Fuchs A, Hilliard J, Davis CG, Bochicchio GV, Southard RE. Polytrauma increases susceptibility to pseudomonas pneumonia in mature mice. Shock. 45:2016.

13. Fukada T, Kato H, Ozaki M, Yagi J. Impact of the timing of morphine administration on lipopolysaccharide-mediated lethal endotoxic shock in mice. Shock. 45:2016.

14. Leelahavanichkul A, Somparn P, Issara-Amphorn J, Elam-ong S, Avihingsanon Y, Hirankarn N, Srisawat N. Serum neutrophil gelatinase associated lipocalin (NGAL) outperforms serum creatinine in detecting sepsis-induced acute kidney injury, experiments on bilateral nephrectomy and bilateral ureter obstruction mouse models. Shock. 45:2016. 